\title{
A Symbolic Framework for the Conformance Checking of Value-Passing Choreographies ${ }^{\star}$
}

\author{
Huu Nghia Nguyen ${ }^{1}$, Pascal Poizat ${ }^{1,2}$, and Fatiha Zaïdi ${ }^{1}$ \\ 1 LRI; Univ. Paris-Sud, CNRS, Orsay, France \\ 2 Univ. Évry Val d'Essonne, Evry, France \\ \{huu-nghia.nguyen, pascal poizat, fatiha.zaidi\}@lri.fr
}

\begin{abstract}
Checking choreography conformance aims at verifying whether a set of distributed peers or local role specifications match a global specification. This activity is central in both top-down and bottomup development processes for distributed systems. Such systems usually collaborate through information exchange, thus requiring value-passing choreography languages and models. However, most of the conformance checking techniques abstract value-passing or bound the domains for the exchanged data. As an alternative, we propose to rely on symbolic models and an extension of the symbolic bisimulation equivalence. This enables one to take into account value passing while avoiding state space explosion issues. Our framework is fully tool supported.
\end{abstract}

Keywords: choreography, specification, conformance, symbolic transition systems, symbolic branching bisimulation, tools.

\section{Introduction}

Context and Issues. A choreography is the description with a global perspective of interactions between roles played by peers (services, organizations, humans) in some collaboration. One key issue in choreography-based development is checking the conformance of a set of local descriptions wrt. the choreography global specification. This issue naturally arises both in bottom-up and in top-down development processes [1], and is also a cornerstone for realizability checking. The definition of conformance should not be too strict. It should support choreography refinement, e.g., with peers and interactions being added in the implementation by the service architect in order to enforce the specification. Finally, entities in a distributed system usually exchange information, i.e., data, while interacting. Consequently, data should be supported in choreography specifications, in the descriptions of the local entities, and in the conformance relation.

Related Work. In Table 1, we compare related conformance checking approaches. Columns 2 and 3 focus on data support. Some approaches [2 [4] $a b$ stract data away. This is known to yield over-approximation issues, e.g., false

\footnotetext{
* This work is supported by the PIMI project (ANR-2010-VERS-0014-03) of the
}

French National Agency for Research. 
Table 1. Choreography conformance approaches

\begin{tabular}{|c|c|c|c|c|c|c|}
\hline & \multicolumn{2}{|c|}{ Data \& Value-Passing } & \multicolumn{2}{|c|}{ Expressiveness } & \multicolumn{2}{|r|}{ Conformance } \\
\hline & supported & treatment & loops & assignment & global & relation (based on) \\
\hline$[2]$ & \multirow{3}{*}{ no } & \multirow{3}{*}{ - } & yes & no & yes & Trace equivalence \\
\hline 3 & & & yes & no & yes & Weak bisimulation \\
\hline (4) & & & yes & no & yes & Strong bisimulation \\
\hline [5] & \multirow{4}{*}{ yes } & closure & yes & yes & no & Weak bisimulation \\
\hline 6 & & closure & no & yes & yes & Branching bisimulation \\
\hline [7] & & bound data & yes & no & yes & Branching bisimulation \\
\hline this paper & & symbolic & yes & limited & yes & Branching bisimulation \\
\hline
\end{tabular}

negatives in the verification process. Data can be supported by working on closed implementation-level systems where sent messages contain only ground data [5, 6]. In such a case, the state space explosion of the system model is limited. However, this is not adequate when working on abstract specifications where there are no such ground sent messages but only free variables and constraints on their values. Another solution is to bound data domains. The issue is that conformance may not yield outside the bounds. Defining bounds in order to avoid false positives in the verification process can be difficult. In our framework, data is supported using a symbolic approach and conformance may be checked for whole data domains.

Columns 4 and 5 are relative to choreography expressiveness. Having both loops and assignments may yield state space explosion if one does not close the system or bound data domains. In this work, we do support loops and a limited form of assignment through message reception.

The last two columns are relative to the kind of conformance being supported and the behavioural equivalence being used. Global conformance is important in conformance checking since one wants not only to know if each peer is conform to its role, i.e., local conformance, but also if the peers altogether have a behaviour that is conform to the choreography. Local conformance does not implies global conformance. Weak and branching bisimulations are able to support internal actions and hiding (formally, $\tau$ actions). This is important, e.g., if one has to deal with messages added to make some choreography realizable. Branching bisimulation [8] has been preferred over weak bisimulation in the last years since it is a congruence, hence supports compositional reasoning.

Symbolic bisimulations, defined on Symbolic Transition Graphs (STGs), have been introduced in [9] with both early and late semantics. In this work, we use a late semantics. STGs have then been extended to STGs with assignments (STGAs) in [10, 11]. These works mostly concentrate on strong and weak bisimulation. Symbolic branching bisimulation has not yet received much attention. As a consequence, there is tool support for symbolic strong bisimulation [12] but not for symbolic branching bisimulation.

Contributions. Our contributions are the following. Based on process algebras for choreography [2, 13], we propose a specification and description language addressing both the global (choreography) and the local (peers description, role requirements) perspective over distributed systems. Our language supports 


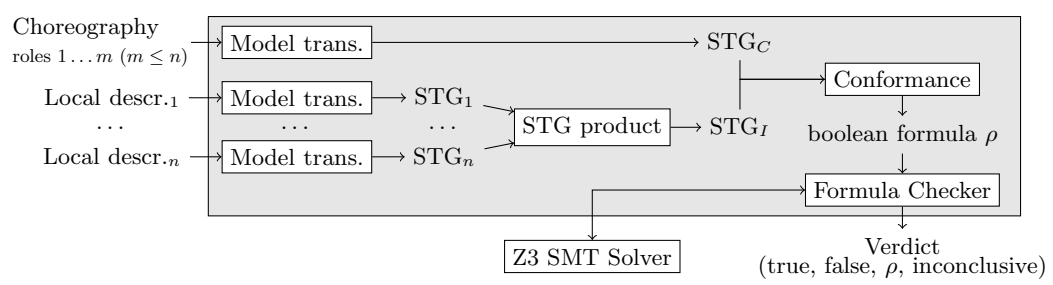

Fig. 1. Architecture of our framework

information exchange and data-related constructs (conditional and loop constructs). We give a fully symbolic semantics to this language using a model transformation into STGs, thus avoiding data abstraction and over-approximation, restriction to manually bound data domains, and limitation to implementationlevel closed descriptions. Accordingly, we build on branching bisimulation [8] and on a symbolic extension of weak bisimulation [11] to develop a specific symbolic version of branching bisimulation dedicated at checking the conformance of a set of local entities wrt. a choreography specification. Our equivalence enables one to check conformance in presence of choreography refinement, i.e., where new peers and/or interactions may be added wrt. the specification. Going further than a true vs. false result for conformance, our approach supports the generation of the most general constraint over exchanged information in order to have conformance. Finally, our framework is fully tool supported 1 .

In the sequel, we present the principles of our approach (technical detail can be found in [14]) and we end with conclusions and perspectives of our work.

\section{Architecture of the Framework}

In this section, we introduce our framework for choreography conformance checking. We also present some of the experiments we have made to evaluate it. The architecture of our framework is given in Figure 1. We take as input a choreography global specification $C$, with $m$ roles. We also take an implementation description $I$, given as $n \geq m$ entity local descriptions. These may correspond either to peer descriptions or to role requirements. The case when $n>m$ denotes, e.g., an implementation where some peers have been added to make a choreography realizable. All inputs are first transformed into STGs. The product of STGs and the restriction to actions in $C$ are used to retrieve a unique STG for $I$, thus yielding two STGs to compare: one for $C(\mathcal{C})$ and one for $I(\mathcal{I})$. We then check if $\mathcal{I}$ conforms to $\mathcal{C}$, which generates the largest boolean formula $\rho$ such that the initial states of $\mathcal{I}$ and $\mathcal{C}$ are conformance related. Finally, this formula is analysed using the Z3 SMT solver 2 in order to reach a conformance verdict. This can be "always true" or "always false", "always" meaning whatever the data values exchanged between peers are. However, sometimes we can have conformance only for a subset of these values. Going further than pure true/false conformance, our framework thus allows

\footnotetext{
${ }^{1}$ Our tool is freely available at http://www.lri.fr/ ${ }^{\sim n h n g h i a / s b b c / ~}$

2 http://research.microsoft.com/en-us/um/redmond/projects/z3/
} 
to compute the largest constraint on data values, $\rho$, that would yield conformance. Complex constraints may cause the solver to return a timeout. In such a case, we emit inconclusiveness as a verdict.

A Language for Choreographies, Roles, and Peers. Since we are interested in an abstract, i.e., implementation independent, formal choreography language, we choose an interaction-based model [15] and the usual $\tau$ actions can be ignored [16]. Our specification language, inspired by [2, 13], is used to specify distributed systems with a global perspective, i.e., choreographies, to define local requirements, i.e., roles, and to describe the pieces of a distributed implementation, i.e., peers. Due to this multi-purpose objective, it is first presented in terms of an abstract alphabet, $A$. We then explain how $A$ can be realized for the different purposes. The syntax of our specification language, $L(A)$, is given by:

$$
L::=1|\alpha| L ; L|L+L| L|L| L[>L|[\phi] \triangleright L|[\phi] * L
$$

A basic activity is either termination (1) or a regular activity $\alpha \in A$. Structuring is achieved using sequencing $(;)$, non deterministic choice $(+)$, parallelism $(\mid)$, and interruption $([>)$. Furthermore, we have data-based conditional constructs, namely guards $(\triangleright)$ and loops $(*)$, where $\phi$ is a boolean expression.

The basis of an interaction-model choreography description is the interaction. Let us denote an interaction $c$ from role $a$ to role $b$ by $c^{[a, b]} . x$, where $x$ is a variable that represents the information exchanged during interaction ( $x$ is omitted when there is none). We stress out that $x$ can be structured, e.g., to denote a multiple data exchange as done in Web services with XML message types. A choreography specification for a set of roles $R$, a set of interactions $C h$, and a set of variables $V$, is an element of $L(A)$ with $A=\left\{c^{[a, b]} . x \mid c \in C h \wedge a \in R \wedge b \in R \wedge a \neq b \wedge x \in V\right\}$.

The events of a local entity $a$ (peer or role) can be abstracted as sending and reception events, denoted respectively with $c^{[a, b]} ! x$ and $c^{[b, a]} ? x$, where $b$ is another entity, i.e., $a \neq b$, and $x$ is the information exchanged during interaction ( $x$ is omitted when there is none). An entity description for an entity $a$, a set of roles $R$ with $a \in R$, a set of interactions $C h$, and a set of variables $V$, is an element of $L(A)$ with $A=\left\{c^{[a, b]} ! x, c^{[b, a]} ? x \mid c \in C h \wedge b \in R \wedge a \neq b \wedge x \in V\right\}$.

Example 1. Let us suppose a shipping choreography between two roles: c (client) and s (shipper). The client first requests shipping by providing the weight of goods to be sent. If this is less than $5 \mathrm{kgs}$ then the goods will be sent for free. Otherwise, the shipping has to be paid. This can be described as follows:

Shipping ::= Request ${ }^{[c, s]} \cdot x_{1} ;\left(\left[x_{1}<5\right] \triangleright\right.$ FreeShip $^{[s, c]}+\left[x_{1} \geq 5\right] \triangleright$ PayShip $\left.^{[s, c]}\right)$ A tentative to implement the shipping choreography is that the client sends the weight to the shipper and then waits for either free or paid shipping, while it is the shipper that checks the weight in order to decide which shipping is used:

$$
\begin{aligned}
& \text { Client } c::=\text { Request }^{[c, s]} ! y_{1} ;\left(\text { FreeShip }^{[s, c]} ?+\text { PayShip }^{[s, c]} ?\right) \\
& \text { Shipper } s::=\text { Request }^{[c, s]} ? z_{1} ;\left(\left[z_{1}<5\right] \triangleright \text { FreeShip }^{[s, c]} !+\left[z_{1} \geq 5\right] \triangleright \text { PayShip }^{[s, c]} !\right)
\end{aligned}
$$

Symbolic Transition Graph. An STG [9] is a transition system where a set of variables, possibly empty, is associated to each state and where each transition 


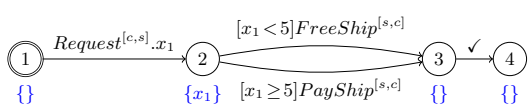

(a) Shipping choreography

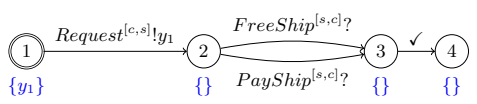

(b) Implementation of Client

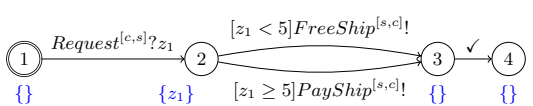

(c) Implementation of Shipper

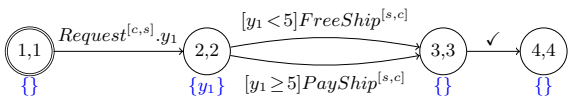

(d) Composition of (b) and (c)

Fig. 2. STGs for Example 1

may be guarded by a boolean expression $\phi$ that determines if the transition can be fired or not. Actions labelling transitions will correspond in our work to the elements of the alphabets we have seen earlier on. We also add a specific event, $\checkmark$, to denote activity termination. A transition from state $s$ to $s^{\prime}$ with a guard $\phi$ and labeled by an action $\alpha$ takes the form $s \stackrel{[\phi] \alpha}{\longrightarrow} s^{\prime}$. We use STGs as a formal model to give semantics to our language. The product of STGs is used to give a semantics to a set of interacting local entities. We assume that the STGs use disjoint sets of variables which can be achieved using, e.g., indexing by the name of the entity. The rule-based of model transformations and our algorithm for the product of STGs can be found in a technical report extension of this paper [14].

Example 2. The STGs for the choreography, the client and shipper in Example1 are shown in Figure 2(a-c). Figure 2(d) presents the product of the STGs in Figure 2(b) and Figure 2(c). The free variables of the states are given below them, e.g., $\left\{x_{1}\right\}$ for state 2 in the choreography STG.

Choreography Conformance. Since our semantic models are STGs, we define conformance over two STGs, $\mathcal{I}$ (implementation) and $\mathcal{C}$ (choreography). We choose branching bisimulation [8] as a basis since it supports equivalence in presence of $\tau$ actions that result from the hiding of interactions added in implementations wrt. specifications, i.e., refinement. However, branching bisimulation is defined over ground terms (no variables), while STGs may contain free variables. In [6, 7], this issue is considered by introducing at each state an evaluation function that maps variables to values, thus reducing open terms to ground ones. This may lead to state space explosion when domains of the variables are big. Alternatively, we base our work on (late) symbolic extensions of bisimulations, introduced in [9 11], that directly support open terms.

To make implementation and specification comparable, we remind the reader that we assume the two STGs have disjoint sets of variables which can be achieved using, e.g., indexing. We also assume that a local entity has the same identifier than the corresponding role in the choreography. This constraint could be lifted using a mapping function. Additional interactions may have been introduced in the implementation wrt. the specification during refinement, e.g., to make it realizable. In order to compare the STGs, we have to hide these interactions. 

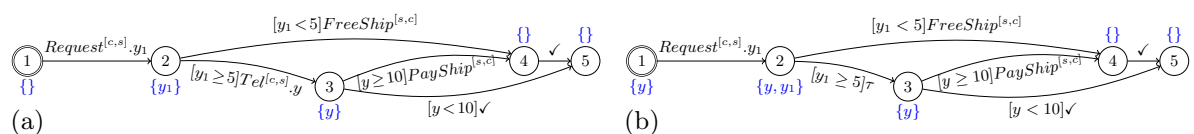

Fig. 3. A refinement (a) for the STG in Figure 2(d) and its restriction (b)

Example 3. We give a refinement example in Figure 31(a) where the client specifies the maximum (s)he agrees to pay for the shipping (y). This influences the sequel of the implementation since the non-free shipping costs $\$ 10$ : if the user requires to pay less, no shipping is done. The restriction of this STG to the set of actions used in the choreography specification, $\left\{\right.$ Request $^{[c, s]}$, FreeShip $^{[s, c]}$, PayShip $\left.^{[s, c]}\right\}$, yields the STG in Figure 3(b) where Tel has been hidden.

Conformance Computation. Our algorithm for the computation of the conformance relation between two STGs [14] is a modification and simplification of the one proposed in [11] that computes symbolic weak bisimulation. Simplification was made possible since there may be $\tau \mathrm{s}$ in $\mathcal{I}$ (after hiding) but not in $\mathcal{C}$. The algorithm outputs a set of boolean formulas $\rho_{s_{1}, s_{2}}$ relative to pairs of states $\left(s_{1}, s_{2}\right), s_{1}$ being in $\mathcal{I}$ and $s_{2}$ in $\mathcal{C}$. $\rho_{s_{1}, s_{2}}$ denotes the conditions under which $s_{1}$ and $s_{2}$ are conformance related. In the algorithm, these boolean formulas are encoded as a Predicate Equation Systems (PES) [10], i.e., a set of functions each of which contains a boolean expression, e.g., $R(x)::=(x \geq 0)$.

Example 4. Applying the algorithm on the STGs in Figure 3(b) (implementation) and in Figure 2(a) (specification), we retrieve the following PES:

$$
\begin{aligned}
& R_{1,1}() \quad::=\forall Z_{0} R_{2,2}\left(Z_{0}, Z_{0}\right) \\
& R_{2,2}\left(y_{1}, x_{1}\right)::=\left(\left(\left(x_{1} \geq 5 \Rightarrow y_{1} \geq 5 \wedge R_{3,2}\left(y_{1}, x_{1}\right)\right) \wedge\left(y_{1} \geq 5 \Rightarrow x_{1} \geq 5 \wedge R_{3,2}\left(y_{1}, x_{1}\right)\right)\right)\right. \\
& \left.\wedge\left(\left(x_{1}<5 \Rightarrow y_{1}<5 \wedge R_{4,3}\right) \wedge\left(y_{1}<5 \Rightarrow x_{1}<5 \wedge R_{4,3}\right)\right)\right) \wedge(\neg(y<10)) \\
& R_{3,2}\left(y_{1}, x_{1}\right)::=\left(\left(x_{1} \geq 5 \Rightarrow y \geq 10 \wedge R_{4,3}\right) \wedge\left(y \geq 10 \Rightarrow x_{1} \geq 5 \wedge R_{4,3}\right)\right) \\
& \wedge\left((\neg(y<10)) \wedge\left(\neg\left(x_{1}<5\right)\right)\right) \\
& R_{4,3}() \quad::=\text { true }
\end{aligned}
$$

Indeed, it can be simplified into $\left\{R_{1,1}::=y \geq 10, R_{2,2}::=y \geq 10, R_{3,2}::=y \geq\right.$ $10 \wedge Z_{0} \geq 5, R_{4,3}::=$ true $\}$ but this demonstrates the need for an automatic PES satisfiability checking procedure as defined below.

PES Satisfiability and Conformance Verdict. The PES resulting from the conformance computation algorithm has to be analyzed in order to reach a conformance verdict. We realize this step with the Z3 SMT Solver by translating the PES into the Z3 input language as demonstrated in Listing 1.1 for the PES in Example 4. Each predicate equation in the PES is translated as a boolean function (using define-fun) and each free variable is translated as an integer function (using declare-fun). We then check R1_1 in order to conclude on conformance. For this, the check-sat Z3 command is run as following. If R1_1 asserted false (as in Listing 1.1) yields an unsat response then there is no interpretation such that $R_{1,1}$ is false, hence we can conclude directly that conformance is true. Otherwise, we have to retry with R1_1 asserted to true to reach a verdict. The result may then be unsat, sat, or timeout corresponding respectively to the conformance being false, may be $(\rho)$, or inconclusive. 
Listing 1.1. Translation into the Z3 language of the PES in Example 4

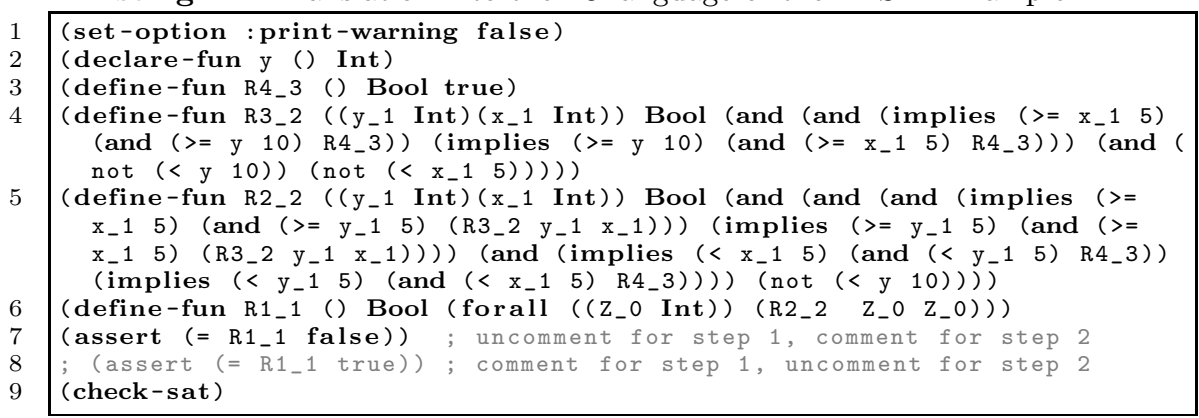

Table 2. Experimental results

\begin{tabular}{|c|c|c|c|c|c|c|c|c|}
\hline \multirow{2}{*}{$\mathrm{Id}$} & \multirow{2}{*}{ Name [Reference] } & \multirow{2}{*}{ \#Peers/Roles } & \multicolumn{2}{|c|}{ Implementation } & \multicolumn{2}{|c|}{ Specification } & \multirow{2}{*}{$\frac{\text { Verdict }}{\text { Orig./Ours }}$} & \multirow{2}{*}{$\begin{array}{l}\text { Duration } \\
\text { (seconds) }\end{array}$} \\
\hline & & & \#Int. & \#Trans./States & \#Int. & \#Trans./States & & \\
\hline \multirow[t]{3}{*}{01} & \multirow[t]{3}{*}{ Shipping [n/a] } & $2 / 2$ & 3 & $4 / 4$ & 3 & $4 / 4$ & -/YES & 0.069 \\
\hline & & $2 / 2$ & 4 & $5 / 5$ & 3 & $4 / 4$ & $-/$ YES & 0.084 \\
\hline & & $2 / 2$ & 4 & $6 / 5$ & 3 & $4 / 4$ & $-/ \rho$ & 0.102 \\
\hline \multirow[t]{2}{*}{$\overline{04}$} & \multirow[t]{2}{*}{ Market [6] } & $4 / 4$ & 8 & $9 / 10$ & 8 & $10 / 10$ & YES/NO & 0.118 \\
\hline & & $8 / 4$ & 16 & $27 / 26$ & 8 & $10 / 10$ & YES/NO & 0.201 \\
\hline 06 & RFQ [7] & $3 / 3$ & 6 & $8 / 7$ & 6 & $8 / 8$ & $\mathrm{NO} / \mathrm{NO}$ & 0.078 \\
\hline 07 & Booking [4] & $4 / 4$ & 8 & $12 / 11$ & 8 & $12 / 11$ & YES/YES & 0.096 \\
\hline
\end{tabular}

Experiments. We have experimented our framework, including on examples from the literature (Tab. 2). For the implementations and the specifications, we respectively give the numbers of peers, roles, interactions, and transitions and states in the corresponding STGs. We also give the conformance verdicts in the paper the example is taken from and with our approach. Finally, we give the execution time (Mac Book Air with OS 10.7, 4 GB RAM, core i5 $1.7 \mathrm{GHz}$ ) for the process described in Figure 1 (but for the time to parse the input files). Rows 1 to 3 correspond to the specification STG in Figure2(a) and, respectively, to the implementations STGs in Figures 2(d) (row 1), 3)(a) (row 2), and 3(b) (row 3 ). Rows 4 and 5 correspond to the example and mutation in 6]. The difference in the verdict comes from the fact the we distinguish between an STG ending with $\checkmark$ (successful termination) or not, hence an implementation deadlocking after achieving all interactions of a specification will not conform to it: the specification may do $\checkmark$ while the implementation may not. Row 6 corresponds to a negative example in [7] and row 7 to a positive one in [4].

\section{Conclusion}

In this paper, we have proposed a formal framework for checking the conformance of a set of role requirements or peer descriptions with reference to a choreography specification. Symbolic models and equivalences enable us to check conformance in presence of data without suffering from state space explosion and without bounding data domains. Going further than strict conformance, we are able to give the most general constraint over data exchanged between peers in order to achieve conformance. Our approach is fully tool supported 1 . 
We advocate that once a choreography projection function supporting data is defined, then our framework could be used not only for conformance checking but also for realizability checking. This is our first perspective. A second perspective is to extend our framework with non-limited assignment and asynchronous communication. Our last perspective is to integrate the extensions of our tools as a verification plugin for the BPMN 2.0 Eclipse editor. A BPMN 2.0 to STG model transformation is ongoing, based on our BPMN to LTS (no data) one [17].

\section{References}

1. Poizat, P.: Formal Model-Based Approaches for the Development of Composite Systems. Habilitation thesis, Université Paris Sud (November 2011), http://www.lri.fr/ poizat/documents/hdr.pdf

2. Qiu, Z., Zhao, X., Cai, C., Yang, H.: Towards the Theoretical Foundation of Choreography. In: Proc. of WWW 2007 (2007)

3. Basu, S., Bultan, T.: Choreography Conformance via Synchronizability. In: Proc. of WWW 2011 (2011)

4. Salaün, G., Bultan, T.: Realizability of Choreographies Using Process Algebra Encodings. In: Leuschel, M., Wehrheim, H. (eds.) IFM 2009. LNCS, vol. 5423, pp. 167-182. Springer, Heidelberg (2009)

5. Li, J., Zhu, H., Pu, G.: Conformance Validation between Choreography and Orchestration. In: Proc. of TASE 2007 (2007)

6. Busi, N., Gorrieri, R., Guidi, C., Lucchi, R., Zavattaro, G.: Choreography and Orchestration Conformance for System Design. In: Ciancarini, P., Wiklicky, H. (eds.) COORDINATION 2006. LNCS, vol. 4038, pp. 63-81. Springer, Heidelberg (2006)

7. Kazhamiakin, R., Pistore, M.: Choreography Conformance Analysis: Asynchronous Communications and Information Alignment. In: Bravetti, M., Núñez, M., Zavattaro, G. (eds.) WS-FM 2006. LNCS, vol. 4184, pp. 227-241. Springer, Heidelberg (2006)

8. Van Glabbeek, R., Weijland, W.: Branching Time and Abstraction in Bisimulation Semantics. Journal of the ACM 43(3) (1996)

9. Hennessy, M., Lin, H.: Symbolic Bisimulations. Theoretical Computer Science 138(2), 353-389 (1995)

10. Lin, H.: Symbolic Transition Graph with Assignment. In: Sassone, V., Montanari, U. (eds.) CONCUR 1996. LNCS, vol. 1119, pp. 50-65. Springer, Heidelberg (1996)

11. Li, Z., Chen, H.: Computing Strong/Weak Bisimulation Equivalences and Observation Congruence for Value-Passing Processes. In: Cleaveland, W.R. (ed.) TACAS 1999. LNCS, vol. 1579, pp. 300-314. Springer, Heidelberg (1999)

12. Basu, S., Mukund,M., Ramakrishnan, C.R., Ramakrishnan,I.V., Verma, R.:Localand Symbolic Bisimulation Using Tabled Constraint Logic Programming. In: Codognet, P. (ed.) ICLP 2001. LNCS, vol. 2237, pp. 166-180. Springer, Heidelberg (2001)

13. Bravetti, M., Zavattaro, G.: Towards a Unifying Theory for Choreography Conformance and Contract Compliance. In: Lumpe, M., Vanderperren, W. (eds.) SC 2007. LNCS, vol. 4829, pp. 34-50. Springer, Heidelberg (2007)

14. Nguyen, H.N., Poizat, P., Zaïdi, F.: A Symbolic Framework for the Conformance Checking of Value-Passing Choreographies. Long version, in P. Poizat Webpage

15. Decker, G., Kopp, O., Barros, A.P.: An Introduction to Service Choreographies. Information Technology 50(2), 122-127 (2008)

16. Kopp, O., Leymann, F.: Do We Need Internal Behavior in Choreography Models? In: Proc. of ZEUS 2009 (2009)

17. Poizat, P., Salaün, G.: Checking the Realizability of BPMN 2.0 Choreographies. In: Proc of SAC 2012 (2012) 